\title{
Human seroprevalence of Borrelia miyamotoi in Manitoba, Canada, in 2011-2014: a cross-sectional study
}

\author{
Kamran Kadkhoda PhD D(ABMM), Cecilia Dumouchel BA, Janna Brancato RN, Ainsley Gretchen BSc, \\ Peter J. Krause MD
}

\section{Abstract}

Background: Hard tick-borne relapsing fever caused by Borrelia miyamotoi has been reported in Russia, the Netherlands, Germany, Japan and the northeastern and upper midwestern United States. We sought to investigate the presence of $B$. miyamotoi infection in humans in Manitoba, Canada.

Methods: Two hundred fifty sera collected from residents of Manitoba with suspected Lyme disease between 2011 and 2014 were tested for Borrelia burgdorferi antibody using a C6 peptide enzyme-linked immunosorbent assay (ELISA) followed by Western blot. Residual sera were then anonymized, stored at $-80^{\circ} \mathrm{C}$ and subsequently thawed and tested for $B$. miyamotoi antibody using a 2-step glycerosphosphodiester phosphodiesterase-based ELISA and Western blot assay.

Results: Twenty-four of the 250 (9.6\%) sera tested positive for B. miyamotoi immunoglobulin G. Participants who were B. miyamotoi seropositive were predominantly male (54\%) and younger on average than those who were seronegative (32 and $44 \mathrm{yr}$ of age, respectively). Participants who were seropositive for $B$. burgdorferi were significantly more likely to be $B$. miyamotoi seropositive than those who were B. burgdorferi seronegative (20.3\% v. 6.6\%, respectively, odds ratio 3.6, 95\% confidence interval $1.5-8.5)$.

Interpretation: This initial report of human B. miyamotoi infection in Canada should raise awareness of hard tick-borne relapsing fever among clinicians and residents of areas in Canada and western North America where Lyme disease is endemic.

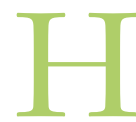
ard tick-borne relapsing fever caused by Borrelia miyamotoi is transmitted by the same hard tick species that transmit the pathogens causing Lyme disease (Borrelia burgdorferi sensu lato), human granulocytic anaplasmosis (Anaplasma phagocytophilum), and human babesiosis (Babesia species). $\cdot^{1-3}$ Hard tick-borne relapsing fever has been reported in Russia, the Netherlands, Germany, Japan and the northeastern and upper midwestern United States. ${ }^{4-14}$ Ixodes ticks infected with $B$. miyamotoi have been described in additional areas, including Canadian provinces. ${ }^{15-18}$ To determine whether human $B$. miyamotoi infection occurs in Canada, we screened for B. miyamotoi antibody in randomly selected archived sera from patients with clinically suspected and antibody-confirmed Lyme disease living in the province of Manitoba, Canada. Study participants were residents of a Lyme disease-endemic region where Ornithodoros ticks that transmit soft tick-borne relapsing fever are not found. ${ }^{19}$

\section{Methods}

\section{Study population}

A total of 250 randomly selected residual sera that were initially submitted to Cadham Provincial Laboratory in Winnipeg, Manitoba, between 2011 and 2014 for Lyme disease serology were tested for B. miyamotoi antibody. The sera were obtained from patients living in Lyme disease-endemic areas in Manitoba with presumed or definite tick exposure (as mentioned on the laboratory request form), and signs and symptoms suggestive of Lyme disease. Manitoba, especially the southern part of the province where these sera were

\section{Competing interests: None declared.}

This article has been peer reviewed.

Correspondence to: Peter Krause, peter.krause@yale.edu

CMAJ Open 2017. DOI:10.9778/cmajo.20170070 
obtained, is a Lyme disease-endemic region with established B. burgdorferi-infected Ixodes scapularis tick populations as well as confirmed human cases (Figure 1). ${ }^{16}$ We only tested sera collected from May through the end of October. Most (83\%) were collected between July and August, which is the season of peak tick activity in Manitoba. We used all sera available from residual serum samples from study participants with positive C6 antibody results. A previous study has shown that patients with $B$. miyamotoi infection may have cross-reacting antibody to B. burgdorferi C6 antigen. ${ }^{8}$ Thus, evaluation of sera from these patients increased our chances of identifying participants infected with $B$. miyamoto $i$ because of crossreacting antibody and because people who have had Lyme disease would have had exposure to I. scapularis ticks that also transmit $B$. miyamotoi. To select a representative sample size for a $5 \%$ margin of error and $95 \%$ confidence interval (CI) with a response distribution of $50 \%$, a sample size of 250 was calculated on the basis of the average of 3000 sera screened per year for Lyme disease in our laboratory since 2011.

\section{Detection of $B$. burgdorferi antibody}

All sera had initially been screened for B. burgdorferi antibody using a C6 peptide enzyme-linked immunosorbent assay (ELISA) kit (Immunetics). Any sera that tested positive with the C6 peptide assay were sent to the National Microbiology Laboratory in Winnipeg, Manitoba, for Lyme disease immunoblot testing. Positive test criteria were based on those of the US Centers for Disease Control and Prevention. ${ }^{20}$ The age and sex of the patients and the specimen collection dates were documented before the specimens were de-identified. Sera were then stored at $-80^{\circ} \mathrm{C}$ for $B$. miyamotoi antibody testing at a later date.

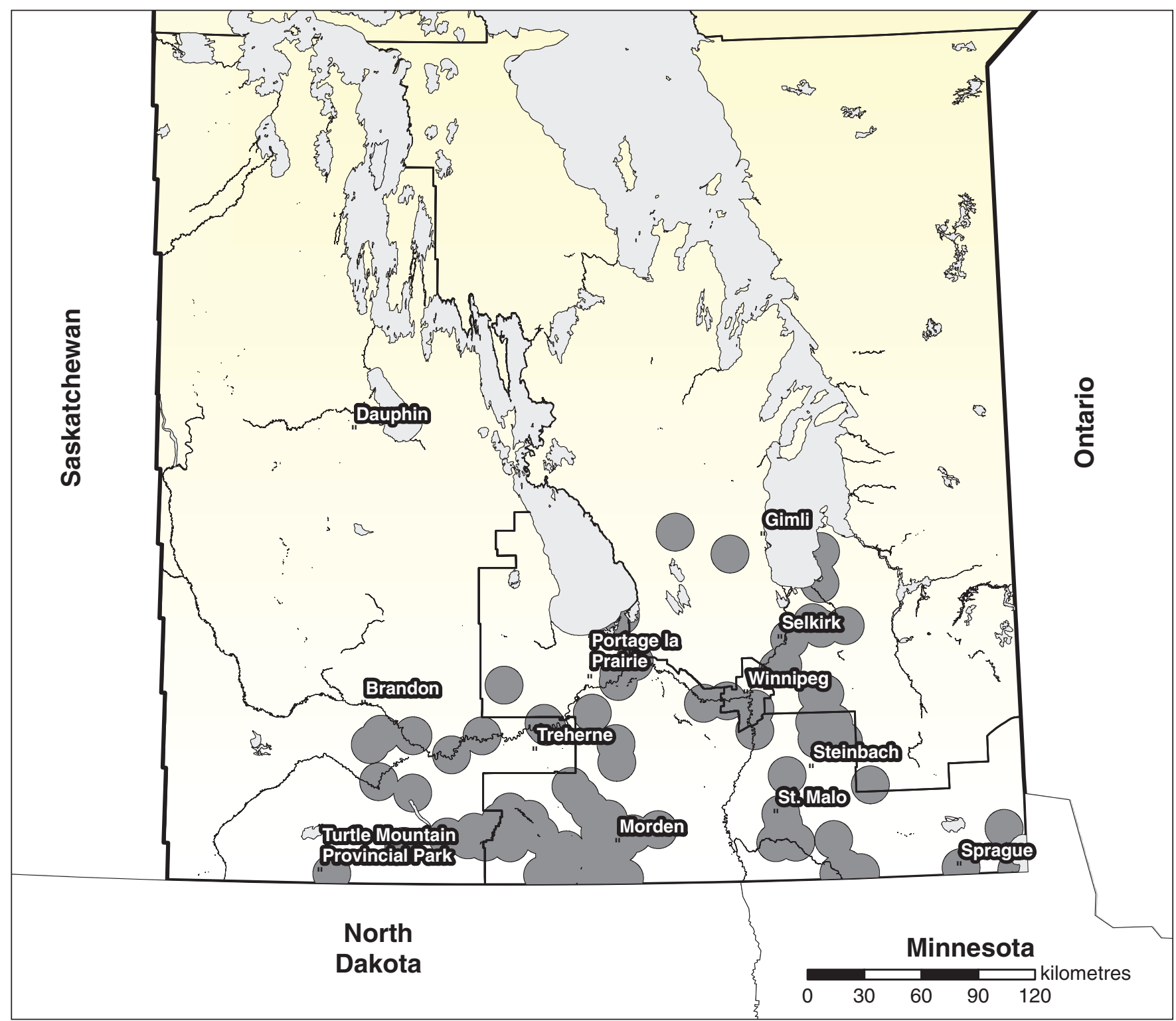

Figure 1: A map of Manitoba, Canada showing Lyme disease risk areas (grey dots) where field surveillance has shown evidence of established blacklegged tick populations between 2006 and 2014. Source: Manitoba Health, Seniors, and Active Living. Published with permission. 


\section{Borrelia miyamotoi glycerophosphodiester phosphodiesterase antibody ELISA}

Borrelia miyamotoi antibody was determined using a 2-tier ELISA assay as previously described. ${ }^{8}$ Serum samples were diluted 1:320 and run in triplicate. Two positive and 3 negative controls were used on each plate. Positive controls consisted of human sera that had been collected from patients with B. miyamotoi infection that was confirmed by polymerase chain reaction (PCR) and that tested positive for B. miyamotoi antibody using both ELISA and Western blot immunoglobulin G (IgG). Negative controls consisted of human sera obtained from 3 healthy New England residents with no history of tick bite or tick-borne disease and who had tested negative for B. miyamotoi by ELISA and Western blot IgG, and by PCR. ${ }^{8} \mathrm{~A}$ sample was considered ELISA positive if the mean optical density value of the sample run in triplicate was equal to or greater than the threshold of 3 standard deviations above the mean of the optical density values of the 3 negative control samples.

\section{Glycerophosphodiester phosphodiesterase Western blot antibody assay}

Sera that tested positive by B. miyamotoi ELISA were then tested by glycerophosphodiester phosphodiesterase (GlpQ) Western blot. ${ }^{8}$ For Western blot, purified rGlpQ (about 250 $\mathrm{ng}$ ) was electrophoresed on each replicate lane of a precast mini sodium dodecyl sulfate electrophoresis gel and transferred to a nitrocellulose membrane. Replicate strips were individually incubated with human serum at a 1:250 dilution in phosphate-buffered saline, washed and incubated with infrared dye (IRdye $800 \mathrm{CW}$ ) goat anti-human IgG (LI-COR Biosciences). Bound antibodies were detected with a LI-COR Odyssey gel-imaging scanner. Samples with a $39-\mathrm{kDa}$ band intensity that approximated that of GlpQ on positive control human serum samples were considered GlpQ antibody positive. Representative Western blots from B. miyamotoi seropositive Manitoba residents with 39-kDa GlpQ bands are shown in Figure 2.

\section{Ethics approval}

We tested de-identified blood specimens; none of the study authors know the names of the patients from whom the blood samples were obtained. The Yale University Human Investigation Committee does not require that approval be obtained for such studies.

\section{Results}

We found serologic evidence of $B$. miyamotoi infection in serum samples from 24 of 250 Manitoba residents with suspected or confirmed Lyme disease. More than twice as many samples were seropositive for B. burgdorferi (22\%) than were

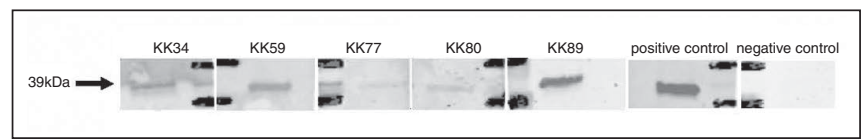

Figure 2: Representative Western blots from Borrelia miyamotoiseropositive Manitoba residents showing 39-kDa glycerophosphodiester phosphodiesterase (GlpQ) bands. seropositive for B. miyamotoi (10\%). Subjects who were B. miyamotoi seropositive were predominantly male (54\%) and were younger on average than those who were seronegative (32 and $44 \mathrm{yr}$ of age, respectively). Participants who were seropositive for $B$. burgdorferi were significantly more likely to be $B$. miyamotoi seropositive than those who were $B$. burgdorferi seronegative $(20.3 \%$ v. $6.6 \%$, respectively, Fisher exact analysis, odds ratio $3.6,95 \%$ CI $1.5-8.5)$.

\section{Interpretation}

To determine whether human B. miyamotoi infection occurs in western North America, we tested archived sera from 250 participants living in Manitoba in a Lyme disease-endemic area and were suspected of having Lyme disease or had confirmed Lyme disease. Twenty-two percent of these residents were seropositive for B. burgdorferi and $10 \%$ were seropositive for B. miyamotoi. As would be expected when 2 pathogens are transmitted by the same I. scapularis tick, the seroprevalence of B. miyamotoi among residents who were $B$. burgdorferi seropositive was greater (more than 3 -fold) than that of residents who were $B$. burgdorferi seronegative. In a group of healthy participants living in a Lyme disease-endemic area of the northeastern US, $9.4 \%$ were B. burgdorferi seropositive and $3.9 \%$ were B. miyamotoi seropositive. ${ }^{8}$ The greater B. miyamotoi seroprevalence in our sample of Canadian participants may be attributed, at least in part, to the fact that they had had recent tick exposure (as evidenced by a suspected or confirmed Lyme disease illness) whereas the American participants were healthy., ${ }^{6,8}$

Tick-borne relapsing fever is the name of a group of diseases caused by Borrelia species that are transmitted by ticks and cause relapsing fever. Most species of Borrelia that cause relapsing fever (such as Borrelia hermsii and Borrelia turicatae) are transmitted by soft ticks, whereas 1 medically important species of relapsing fever Borrelia is transmitted by hard-bodied ticks (B. miyamotoi). The risk of exposure is variable. Seroprevalence studies in New England suggest that $B$. miyamoto $i$ infection may be as common as anaplasmosis and babesiosis. Borrelia miyamotoi has been found in I. scapularis ticks in all Canadian provinces except Newfoundland and Labrador, and human cases are likely to be found wherever Lyme disease is endemic. ${ }^{16}$ Borrelia miyamotoi may cause serious complications, including meningoencephalitis, in immunocompromised hosts. ${ }^{5,714} \mathrm{~A}$ quarter of the cases in 1 large B. miyamotoi case series were admitted to hospital. ${ }^{10}$ Several studies suggest that $B$. miyamotoi may be transmitted through blood transfusion, consistent with the high levels of spirochetemia that occur with Borrelia species that cause relapsing fever. ${ }^{21}$

The general clinician practising in areas where this infection is found in ticks should be aware that a febrile illness without an erythema migrans rash (and especially a recurrent febrile illness) in late spring, summer or early autumn may be due to $B$. miyamotoi. Patients infected with $B$. miyamotoi may test positive with the $B$. burgdorferi C6 ELISA antibody assay and the B. burgdorferi Western blot assay. ${ }^{8}$ Diagnosis can be confirmed by identification of spirochetes on blood smear or B. miyamotoi PCR, or a 4-fold rise in B. miyamoto $i$ antibody in acute and convalescent sera. Serological testing for B. miyamo- 
to $i$ is currently unavailable in Canada. We hope that our report will accelerate the availability of such testing. Polymerase chain reaction testing for B. miyamotoi is available at the National Microbiology Laboratory. This test is especially useful when patients are experiencing acute illness and when antibiotic treatment has not been started. The PCR assay can be performed on both whole blood and cerebrospinal fluid specimens. Patients infected with $B$. miyamotoi should be treated in the same manner as patients with Lyme disease.

\section{Limitations}

We used B. miyamotoi GlpQ as antigen for our ELISA and Western blot antibody assays. Glycerophosphodiester phosphodiesterase is not produced by Lyme disease spirochetes and therefore B. burgdorferi-specific antibodies will not cross-react with B. miyamotoi GlpQ antigen. ${ }^{22}$ In contrast, all Borrelia species that cause relapsing fever produce GlpQ, so it is possible that some of the participants living in Manitoba who reacted against B. miyamotoi GlpQ antigen might previously have been infected by species that cause soft tick-borne relapsing fever such as $B$. hermsii or $B$. parkeri rather than B. miyamotoi $i^{8,22}$ This is unlikely because the nearest areas in Canada where soft tick-borne relapsing fever is endemic are in British Columbia, which is more than $1500 \mathrm{~km}$ from Manitoba, and the incidence of soft tick-borne relapsing fever there is low, although travel history was not assessed. ${ }^{19}$ Furthermore, had these patients been infected with soft tick-borne relapsing fever, there would not have been a strong correlation between B. burgdorferi and B. miyamotoi antibodies, as was observed in our study participants. Certain gram-negative bacterial species such as Serratia marcescens and Hemophilus influenzae produce GlpQ. ${ }^{8,22}$ The evolutionary distance between these organisms and $B$. miyamoto $i$ is wide, however, and antibodies against these bacteria would not be expected to cross-react with $B$. miyamoto $i$ antibody, although this has not been tested. Furthermore, cross-reacting antibody against bacteria not transmitted by ticks would not explain the association between the B. burgdorferi and B. miyamotoi antibodies.

\section{Conclusion}

We described initial evidence of human B. miyamotoi infection in Canada. Because B. miyamotoi infection may become a substantial health problem, additional studies of human $B$. miyamotoi infection in Canada with larger sample numbers and sampling of the general population will help provide a better understanding of the health burden of this recently discovered tick-borne disease. Such information should raise awareness of hard tick-borne relapsing fever among clinicians and residents of areas in Canada were Lyme disease is endemic.

\section{References}

1. Fukunaga M, Takahashi Y, Tsuruta Y, et al. Genetic and phenotypic analysis of Borrelia miyamotoi sp. nov., isolated from the ixodid tick Ixodes persulcatus, the vector for Lyme disease in Japan. Int 7 Syst Bacteriol 1995;45:804-10.

2. Scoles GA, Papero M, Beati L, et al. A relapsing fever group spirochete transmitted by Ixodes scapularis ticks. Vector Borne Zoonotic Dis 2001;1:21-34.

3. Barbour AG, Bunikis J, Travinsky B, et al. Niche partitioning of Borrelia burgdorferi and Borrelia miyamoto $i$ in the same tick vector and mammalian reservoir species. Am 7 Trop Med Hyg 2009;81:1120-31.
4. Platonov AE, Karan LS, Kolyasnikova NM, et al. Humans infected with relapsing fever spirochete Borrelia mivamotoi, Russia. Emerg Infect Dis 2011;17:1816-23.

5. Gugliotta JL, Goethert HK, Berardi VP, et al. Meningoencephalitis from Borrelia miyamotoi in an immunocompromised patient. N Engl F Med 2013;368:240-5.

6. Krause PJ, Narasimhan S, Wormser GP, et al. Human Borrelia miyamoto infection in the United States. N Engl 7 Med 2013;368:291-3.

7. Hovius JW, de Wever B, Sohne M, et al. A case of meningoencephalitis by the relapsing fever spirochaete Borrelia miyamotoi in Europe. Lancet 2013;382:658.

8. Krause PJ, Narasimhan S, Wormser GP, et al.; Tick Borne Diseases Group. Borrelia miyamotoi sensu lato seroreactivity and seroprevalence in the northeastern United States. Emerg Infect Dis 2014;20:1183-90.

9. Jobe DA, Lovrich SD, Oldenburg DG, et al. Borrelia miyamotoi infection in patients from Upper Midwestern United States, 2014-2015. Emerg Infect Dis 2016;22:1471-3

10. Molloy PJ, Telford SR III, Chowdri HR, et al. Borrelia miyamotoi disease in the United States: a case series. Ann Intern Med 2015;163:91-8.

11. Sato K, Takano A, Konnai S, et al. Human infections with Borrelia miyamotoi, Japan. Emerg Infect Dis 2014;20:1391-3.

12. Wagemakers A, Staarink PJ, Sprong H, et al. Borrelia miyamotoi: a widespread tick-borne relapsing fever spirochete. Trends Parasitol 2015;31:260-9.

13. Krause PJ, Fish D, Narasimhan S, et al. Borrelia miyamotoi infection in nature and in humans. Clin Microbiol Infect 2015;21:631-9.

14. Boden K, Lobenstein S, Hermann B, et al. Borrelia miyamotoi-associated neuroborreliosis in immunocompromised person. Emerg Infect Dis 2016;22:1617-20.

15. Mun J, Eisen RJ, Eisen L, et al. Detection of a Borrelia miyamotoi sensu lato relapsing-fever group spirochete from Ixodes pacificus in California. $7 \mathrm{Med}$ Entomol 2006;43:120-3.

16. Dibernardo A, Cote T, Ogden NH, et al. The prevalence of Borrelia miyamotoi infection, and co-infections with other Borrelia spp. in Ixodes scapularis ticks collected in Canada. Parasit Vectors 2014;7:183.

17. Padgett K, Bonilla D, Kjemtrup A, et al. Large scale spatial risk and comparative prevalence of Borrelia miyamotoi and Borrelia burgdorferi sensu lato in Ixodes pacificus. PLoS One 2014;9:e110853.

18. Salkeld DJ, Nieto NC, Carbajales-Dale P, et al. Disease risk and landscape attributes of tick-borne Borrelia pathogens in the San Francisco Bay Area, California. PLoS One 2015;10:e134812.

19. Lindquist EE, Galloway TD, Artsob H, et al. A bandbook to the ticks of Canada (Ixodida: Ixodidae, Argasidae). Monograph Series No. 7. Biological Survey of Canada: 2016. Available: http://biologicalsurvey.ca/public/Bsc/Controller/ Page/AGR-001-Ticks-Monogram.pdf (accessed 2017 June 27).

20. Lyme disease: two-step laboratory testing process. Atlanta: Centers for Disease Control and Prevention; 2015. Available: www.cdc.gov/lyme/diagnosistesting/ LabTest/TwoStep/index.html (accessed 2017 June 27).

21. Krause PJ, Hendrickson J, Steeves T, et al. Blood transfusion transmission of the tick-borne relapsing fever spirochete Borrelia miyamotoi in mice. Transfusion 2015;55:593-7.

22. Schwan TG, Schrumpf ME, Hinnebusch BJ, et al. GlpQ: an antigen for serological discrimination between relapsing fever and Lyme borreliosis. 7 Clin Microbiol 1996;34:2483-92

Affiliations: Cadham Provincial Laboratory (Kadkhoda, Gretchen); Departments of Medical Microbiology \& Infectious Diseases and Immunology (Kadkhoda), Max Rady College of Medicine, Rady Faculty of Health Sciences, University of Manitoba, Winnipeg, Man.; Yale School of Public Health (Dumouchel, Brancato, Krause); Yale School of Medicine (Krause), New Haven, CT

Contributors: Kamran Kadkhoda and Peter Krause made substantial contributions to the conception and design of the work. Kamran Kadkhoda worked with the Manitoba Department of Health, Seniors and Active Living to obtain the epidemiological information in the form of the Figure 1 map. Janna Brancato helped with specimen processing. All authors helped with acquisition, analysis and interpretation of data, revised the manuscript critically for important intellectual content, provided final approval of the version to be published, and agreed to be accountable for all aspects of the work in ensuring that questions related to the accuracy or integrity of any part of the work are appropriately investigated and resolved.

Funding: This work was supported by the National Institutes of Health (1R56AI114859-01), and the Gordon and Llura Gund Foundation.

Acknowledgements: We thank Dr. Sukanya Narasimhan and Dr. Erol Fikrig for providing us with $B$. miyamotoi $\mathrm{rGlpQ}$ antigen. We also thank Scott Graham-Derham for providing the map for Figure 1 and Nathalie Lavoie and Morgan Smith for Figure 2.

Supplemental information: For reviewer comments and the original submission of this manuscript, please see www.cmajopen.ca/content $/ 5 / 3 /$ E690/suppl/DC1. 